Arora et al. (2019) Repertoire-Based Diagnostics Using Statistical Biophysics

\title{
1 Repertoire-Based Diagnostics Using Statistical Biophysics
}

2 Rohit Arora, $\mathrm{PhD}^{1}$, Joseph Kaplinsky, $\mathrm{PhD}^{2}$, Anthony Li, MS, ${ }^{1}$ and Ramy Arnaout, MD, DPhil ${ }^{1,3 *}$

$3{ }^{1}$ Division of Clinical Pathology, Department of Pathology, Beth Israel Deaconess Medical Center,

4 Boston, MA 02215

$5{ }^{2}$ Department of Health Technology, DTU Healthtech, Technical University of Denmark, Produk-

6 tionstorvet, 2800, Kongens Lyngby, Denmark.

$7{ }^{3}$ Harvard Medical School, 25 Shattuck St, Boston, MA 02115

8 *To whom correspondence should be addressed at rarnaout@gmail.com 
Arora et al. (2019) Repertoire-Based Diagnostics Using Statistical Biophysics

\section{Abstract}

10 A fundamental challenge in immunology is diagnostic classification based on repertoire se-

11 quence. We used the principle of maximum entropy (MaxEnt) to build compact representations

12 of antibody $(\mathrm{lgH})$ and T-cell receptor (TCR 3$)$ CDR3 repertoires based on the statistical biophysi-

13 cal patterns latent in the frequency and ordering of repertoires' constituent amino acids. This

14 approach results in substantial advantages in quality, dimensionality, and training speed com-

15 pared to MaxEnt models based solely on the standard 20-letter amino-acid alphabet. De-

16 scriptor-based models learn patterns that pure amino-acid-based models cannot. We demon-

17 strate the utility of descriptor models by successfully classifying influenza vaccination status

$18\left(A \cup C=0.97, p=4 \times 10^{-3}\right.$ ), requiring only 31 samples from 14 individuals. Descriptor-based MaxEnt

19 modeling is a powerful new method for dissecting, encoding, and classifying complex reper-

20 toires. 

aCC-BY-NC 4.0 International license.

Arora et al. (2019) Repertoire-Based Diagnostics Using Statistical Biophysics

\section{Introduction}

A major challenge in systems immunology is determining how to describe the sequence-level heterogeneity of antibody (immunoglobulin; Ig) and T-cell receptor (TCR) repertoires in ways that facilitate the identification of meaningful patterns. Sequence-frequency distributions-for example, counts of unique IgH or TCR $\beta$ CDR3s - are commonly used but not ideal for interpersonal comparisons, since repertoires from different people are largely disjoint (Robins et al., 2010; Arnaout et al., 2011). Motif-frequency distributions, which count how often each of the $20^{n}$ possible $n$-mers appears in a repertoire (for some choice of $n$ ), are more likely to overlap between individuals, but may fail to detect probabilistic or higher-order patterns and are subject to sampling-related bias unless $n$ is small. Comparisons of frequency distributions between repertoires from different individuals have yielded important insights (Parameswaran et al., 2013; Kaplinsky et al., 2014; Emerson et al., 2017; Sun et al., 2017) but the limitations of this approach suggest a need for complementary methods. One such method is maximum-entropy (MaxEnt) modeling (Fig. 1).

MaxEnt models, which were first developed for statistical physics and information theory (Jaynes, 1957), can be used to describe repertoires (or other complex ensembles of proteins, nucleic acids, etc.) in terms of constraints called biases that determine the ways in which a given repertoire differs from a uniform distribution of sequences (Yeo and Burge, 2004; Russ et al., 2005; Seno et al., 2008; Mora et al., 2010; Marks et al., 2011). Given a set of features-for example, the frequencies of the 20 amino acids and the $20^{2}=400$ nearest-neighbor amino-acid pairs ("neighbors" being defined as contiguous N-to-C-terminus amino acids) - a MaxEnt model describes the degree to which each feature is biased away from its value in a uniform repertoire, taking all the other biases into account. For example, the bias for the pair cysteine-alanine (CA) describes the extent to which the frequency of CA in the repertoire differs from what would be expected given the frequencies of the individual amino acids $C$ and $A$, the pairs $X C$ and $A X$ (for all amino acids $X)$, and so on. MaxEnt models deconvolute the hundreds or thousands of interactions among features into separate components, which then together govern the generation of the observed sequence- and motif-frequency distributions. Thus MaxEnt models can be thought of as capturing the underlying generative structure of the repertoire.

MaxEnt modeling of $\operatorname{lgH}$ and TCR $\beta$ CDR3s, as well as of other protein families, has shown that the frequencies of a single set of neighboring amino-acid pairs capture a remarkable amount of information (Russ et al., 2005; Seno et al., 2008; Mora et al., 2010; Marks et al., 2011), but not 
Arora et al. (2019) Repertoire-Based Diagnostics Using Statistical Biophysics

53

all of it (Bialek and Ranganathan, 2007). Additional sets of pairs-for example, second-, third-, or fourth-nearest neighbors (Mora et al., 2010)_add precision but at the cost of a substantial increase in the number of model parameters ( 400 per set of pairs). This increase can affect the coverage per feature (the total number of instances of the features in the sample divided by the number of features), model quality and interpretability, and training time. The root of the problem is that the amino-acid alphabet has 20 letters: as a result, parameters, data, and computational requirements scale roughly as powers of 20 . The alphabet also causes a second important problem: letters in and of themselves, while a familiar and useful shorthand, lack information about similarities and differences among the multi-faceted biophysical entities they representthe amino acids - for example, that $A$ is more like glycine $(G)$ than tyrosine $(Y)$-that may well contain meaningful patterns that are not obvious from, or captured by, the shorthand alone.

These two problems can be addressed simultaneously by swapping the traditional amino-acid alphabet for a smaller set of descriptors derived from amino acids' physicochemical properties, especially for pairs and higher-order associations (e.g. consecutive triples) (Fig. 1a). Over two dozen lipophilic (e.g., hydrophobicity), steric (e.g. molecular weight) and electrical (e.g. charge) properties have been precisely measured (Sandberg et al., 1998; Kim et al., 2016). These properties have been shown to correlate with each other, such that the first few principal components (PCs) explain a majority of the overall variance (Hellberg et al., 1987; Sandberg et al., 1998). These PCs are natural candidates for a reduced alphabet: they define orthogonal dimensions of a continuous space in which the discrete amino acids are embedded (Fig. 1a). Whereas in "letter space" there is no concept of distance between amino acids, in "descriptor space" amino acids with similar properties are closer together (e.g., with $A$ nearer $G$ than $Y$ ) (Fig. 1b). Such embeddings have been explored in immune-repertoire analysis (Greiff et al., 2017; Ostmeyer et al., 2017, 2019) and other contexts (Dosztányi and Torda, 2001; Walter et al., 2005; Susko and Roger, 2007; Stephenson and Freeland, 2013). We investigated whether descriptor-based MaxEnt models of IgH and TCR $\beta$ CDR3 repertoires could improve on models based on amino acids alone by allowing more data per parameter (less sampling error), shorter training time, and better interpretability (Fig. 1c-d), in principle leading to better models useful for classification of states of health and disease. 
Arora et al. (2019) Repertoire-Based Diagnostics Using Statistical Biophysics

82

83

84

85

86

87

88

89

90

91

\section{Results}

Using 26 measurements carried out on the 20 standard amino acids, we derived five biophysical descriptors that together explained $92 \%$ of the variance in amino acids' physicochemical properties. Each descriptor is a PC, i.e. a linear combination of the measurements. The first three descriptors corresponded roughly to surface area/chromatographic properties (explaining $41 \%$ of the overall variance), van der Waals volume (25\%), and charge (14\%) and together explained $79 \%$ of variance, an increase over the $68 \%$ previously reported for the first three descriptors derived from measures of both the standard and additional non-canonical amino acids (Sandberg et al., 1998).

We trained amino-acid- and descriptor-based MaxEnt models on representative IgH and TCR $\beta$ CDR3 repertoires (Fig. 2) and asked which type of model better described test sets of CDR3 sequences set aside from each repertoire, using a nearest- and next-nearest-neighbor aminoacid model as the benchmark (Methods) (Mora et al., 2010). We compared this benchmark to two descriptor models: one that fit similar positional information but with fewer parameters, and one that fit more positional information with a more similar number of parameters. To score these comparisons, we calculated the (logarithm of the) relative probability that each sequence $\sigma$ in the relevant test set belonged to its repertoire according to each of the two models $\left(M_{d}\right.$, descriptor model; $M_{a}$, amino-acid model):

$$
\ln \frac{p\left(\sigma \mid M_{d}\right)}{p\left(\sigma \mid M_{a}\right)}
$$

and calculated the percent of sequences for which each model was a better fit. A score of $100 \%$ for a given model meant that that model gave a higher probability for every sequence in the test set. As validation, we confirmed that $\mathrm{lgH}$ models scored $>99 \%$ of $\mathrm{IgH}$ sequences better than TCR $\beta$ models (Fig. 3a, left), and TCR $\beta$ models scored $>99 \%$ of TCR $\beta$ sequences better than IgH models (Fig. 3a, right).

Test 1: Similar positional information. We first compared models that incorporated similar positional information: single-amino-acid positions and nearest- and next-nearest neighbor pairs (see Methods). The amino-acid models required $2 \times 20^{2}=800$ parameters to capture the pairwise information vs. just $2 \times 5^{2}=50$ parameters for the descriptor models (for each of $\operatorname{lgH}$ and TCR $\beta$ ). We predicted that amino-acid models would outperform descriptor models on this test, since for every pair of positions the amino-acid model should have a slight edge, given that descriptors 
Arora et al. (2019) Repertoire-Based Diagnostics Using Statistical Biophysics

111

112

113

114

115

116

117

118

119

120

121

122

123

124

125

126

127

128

129

130

131

132

133

134

135

136

137

138

139

140

141

142

capture only $92 \%$ of the variance in amino acids' biophysical properties. Thus this test was expected to provide an estimate of the cost of swapping alphabets. As predicted, amino-acid models outperformed descriptor models, by a wide margin: $94.2 \%$ to $5.8 \%$ for IgH (Fig. $3 \mathrm{~b}$, left) and $99.6 \%$ to $0.4 \%$ for TCR $\beta$ (Fig. 3b, right). The median sequence had a probability that was $\sim 240(\operatorname{lgH})$ and $\sim 87,000($ TCR $\beta)$ times as high according to the amino-acid model as according to the descriptor model. For the amino-acid models, sequences from the final samples often contained the canonical CDR3 stems (see Discussion), but these were rare for final samples from these simple descriptor models.

Test 2: Similar numbers of parameters. A primary motivation for developing descriptor models is their ability to capture information at a given set of positions with fewer parameters than aminoacid models; the corollary is that for a given number of parameters, descriptor models can capture more positional information. Specifically, for the 400 parameters amino-acid models require to capture information about nearest-neighbor pairs, descriptor models can also capture information about next-nearest-neighbor pairs and cross-loop (Buck, 1992; Weitzner et al., 2015) pairs, both for the stem (or "torso;" see Methods) and the entire CDR3, as well as about consecutive three-amino-acid motifs ( $n=325$ non-length parameters for descriptor models vs. 420 for amino-acid models, including the 20 single-amino-acid biases). We therefore first compared 420-parameter amino acid models against 325-parameter descriptor models that fit this additional information.

We expected the descriptor models to outperform these amino-acid models, which, unlike our benchmark amino-acid models, did not fit next-nearest-neighbor pairs, reflecting the utility of additional positional constraints for defining CDR3s. We found that descriptor models outperformed amino-acid models handily, with scores of $85.6 \%$ to $14.4 \%$ for $\mathrm{lgH}$ (Fig. 3c, left) and $86.9 \%$ to $13.1 \%$ for TCR $\beta$ (Fig. 3c, right). The median sequence in the test set was 217 - and $82-$ fold more likely to have been produced by the descriptor model for IgH and TCR $\beta$, respectively. More remarkably, descriptor models also outperformed our benchmark amino-acid models, even though the descriptor models had less than half the parameters (820 vs. 325 non-length parameters), by almost the same margin for $\mathrm{lgH}, 80.7 \%$ to $19.3 \%$ (Fig. 3d, left), but by much less for TCR $\beta$, at $54.6 \%$ to $45.4 \%$ (Fig. 3d, right), leaving the main advantages in this case being coverage and training time. In contrast to the amino-acid models, the familiar start and end motifs (see Discussion) had already been learned in just a few iterations/minutes, requiring just a few hundred sample sequences on which to learn. 
Arora et al. (2019) Repertoire-Based Diagnostics Using Statistical Biophysics

143 Test 3: Classification. Finally, we sought to test the utility of descriptor models in distinguishing

144 between states of health. As proof of principle, we fit descriptor models on 31 before-and-after

$145 \mathrm{IgG}^{+}$repertoires (including three replicates) from 14 healthy human volunteers who were admin-

146 istered a seasonal trivalent influenza vaccine (Vollmers et al., 2013). We had previously shown

147 that vaccination leads to prominent changes in both repertoires' raw and functional diversity

148 (Arora et al., 2018), but withheld diversity measurements from the present study in order to test

149 the discriminatory power of the models in the absence of that additional information. Using strat-

150 ified 3-fold cross-validation, we found that descriptor models distinguished between pre- and

151 post-vaccination pairs with median AUC of 0.97 ( $p=4 \times 10^{-3}$; Fig. 4). It is worth noting that apply-

152 ing PCA to the models to reduce them to two dimensions, failed to distinguish between day 0

153 and day 7 , consistent with a lack of necessity for directions of greatest variance to correlate with

154 differences in states of health.

\section{Discussion}

156 MaxEnt is a powerful method for modeling highly complex systems such as $\operatorname{lgH}$ and TCR $\beta$ rep-

157 ertoires but exhibits practical limitations related to speed and dimensionality when fit on amino

158 acids using only the standard 20-letter alphabet. Here we demonstrate significant advantages

159 by fitting on biophysical descriptors. We show that appropriate descriptor models can capture

160 more of the information in the repertoire with fewer parameters, and that they can successfully

161 classify health-based states with high accuracy, using the $\operatorname{lgG}^{+} \mathrm{B}$-cell response to influenza vaccination as proof of principle.

163

164

165

166

167

168

169

170

171

172

173

174

A key finding was that descriptor models outperformed amino acid models only once additional positional information was included; when fit on similar positional information-single/overall frequencies and nearest- and next-nearest neighbors-amino-acid models performed better. This finding raises the question of what the relative contributions are of the additional types of positional information fit by the winning descriptor models. There were three additional types of positional information beyond nearest-neighbors: parameters for the stem, cross-pairs, and triples. We chose to fit the stem explicitly because the first and last few amino acids in CDR3s of both $\lg \mathrm{H}$ and TCR $\beta$ are stereotypical, almost canonically beginning with a cysteine (excluded in some definitions), followed by a hydroxylic or small aliphatic amino acid (most often glycine, alanine, or threonine) at the second position, and a basic amino acid (arginine/lysine) at the third position and ending with a methionine or phenylalanine, followed by an aspartate, then valine or tyrosine, and finally tryptophan for $\operatorname{lgH}$, and starting with cysteine, alanine, and a pair of hydrox- 
Arora et al. (2019) Repertoire-Based Diagnostics Using Statistical Biophysics

175

176

177

178

ylic or basic amino acids and often ending with glutamate, a variable amino acid, and two aromatic amino acids for TCR $\beta$. These amino acids are important in establishing the stem-loop (or "torso-head") configuration of CDR3s (Buck, 1992; Weitzner et al., 2015). In IgH and TCR $\beta$ the stem is most often encoded by the end of the $\mathrm{V}$ gene segment and start of the $\mathrm{J}$, not by the highly variable D gene segment and adjacent non-templated nucleotides (Lefranc et al., 1999); fitting the stem may be allowing the remaining parameters to better fit the more variable region. Fitting cross pairs - the product of descriptor values at the first and last amino acids, second and second-to-last, etc. - was similarly inspired by CDR3s' stem-loop architecture and may be having a similar benefit. Amino-acid triples are important parts of binding motifs and have been shown to have discriminatory power in IgH in model systems (Sun et al., 2017); it is reasonable that the biophysical patterns they represent also add resolving/discriminatory power. A systematic dissection of these contributions is left for future work.

A further finding was the disparity between the performance of the top descriptor model for $\lg \mathrm{H}$, relative to the benchmark amino-acid model, vs. that for TCR $\beta$ : the descriptor model scored $80.7 \%$ for $\mathrm{lgH}$ vs. only $54.6 \%$ for TCR $\beta$. A value over $50 \%$ indicates that the descriptor model is capturing more information than the amino-acid model, but in the case of TCR $\beta$, the benefit was modest. We considered three possible explanations. First, it is possible that both models captured substantially all of the information present in the training set; however, had this been the case, the models' final samples would likely have been nearly identical to the training set, and they were not. Second, the additional information in the TCR $\beta$ repertoire may not be well captured by the additional positional relationships fit by these models (stem, cross-pairs, triples), but may reside instead in some other relationship(s). Third, the modest benefit may mean that there are isolated (i.e. discontinuous) probability densities in this training set, which the Markov chain used to generate samples (Fig. 1c) has difficulty navigating (van Ravenzwaaij et al., 2018). If so, it may be that somatic hypermutation in the $\lg \mathrm{H} C D R 3$ s bridges probability densities in $\lg \mathrm{H}$ repertoires that in TCR $\beta$ repertoires, which lack somatic hypermutation, remain separate. Conversely, the greater improvement noted for IgH may reflect descriptor models' ability to detect biophysical similarities among these related sequences, which may be less prominent in TCR $\beta$ repertoires but simultaneously difficult to capture in amino-acid models.

The success of descriptor models in correctly discriminating between pre- and post-influenza vaccination suggests potential medical applications. We note that vaccination, like many immunological perturbations, results in systems- as well as sequence-level changes; for example, changes in immunological/repertoire diversity (Jiang et al., 2013; Vollmers et al., 2013). We pre- 
Arora et al. (2019) Repertoire-Based Diagnostics Using Statistical Biophysics

208 viously showed that the combination of raw and functional diversity, measured with various fre-

209 quency weightings, can discriminate between pre- and post-vaccination sample pairs with high

210 accuracy, likely in part by detecting clonal expansion with selection (Arora et al., 2018). Howev-

211 er, changes in diversity, while potentially useful as part of a screening test, are not sufficiently

212 specific to serve as a general diagnostic modality. The present study shows that even without

213 the powerful discriminatory information that diversity adds, descriptor models are capable of

214 highly sensitive and specific diagnostic discrimination, with high AUC and low p-value from

215 small numbers of subjects and samples. The relatively small number of parameters and these

216 parameters' relatively straightforward interpretability (compared to, for example, parameters in

217 deep-learning models) suggest that leveraging the statistical biophysics of repertoires' amino-

218 acid composition is a promising direction for dissecting immune responses for diagnostic and

219 therapeutic purposes. This method is extensible to more or all of IgH or TCR $\beta$, to the comple-

220 mentary chain $(\mathrm{lgL} / \mathrm{TCR} \alpha)$, and indeed to other proteins or biopolymers, leveraging the power of

221 functional relationships to shrink alphabets while increasing their information density.

\section{Acknowledgements}

223 This work used the resources allocated via Jetstream cloud service (allocation ID: TG-

$224 \mathrm{BIO170094)}$ ) of Extreme Science and Engineering Discovery Environment (XSEDE), which is 225 supported by National Science Foundation grant number ACl-1548562. Research Computing 226 Group at the High Performance Computing Cluster at Harvard Medical School. The authors 227 would like to thank Dr. Mohammed Al-Quraishi for helpful discussion.

\section{Methods}

229

230

231

232

233

234

235

236

237 238 90:10 into training and test sets; for Test 3 all sequences were used.

Descriptors. Twenty-six biophysical measurements were previously made on a set of 87 amino acids, which included the standard 20 (Sandberg et al., 1998). We filtered out non-standard amino acids and applied PCA to the standard 20 amino acids (using Python; sklearn.decomposition.PCA library). The top five PCs, which together explained $92 \%$ of the observed variance, were each normalized to a mean of 0 and maximum range $[-1,1]$ and used as biophysical descriptors.

Data. IgG (Vollmers et al., 2013), memory IgH (DeWitt et al., 2016), and TCR (Emerson et al., 2017) CDR3 repertoires were obtained and processed as previously described (Arora et al.,

2018). For each dataset in Tests 1 and 2, 500,000 sequences were chosen at random and split 
Arora et al. (2019) Repertoire-Based Diagnostics Using Statistical Biophysics

239 Models. MaxEnt models were trained on features' expectation values, with one parameter per

240 feature (the bias). For Tests 1 and 2, amino-acid models were trained on the observed frequen-

241 cies of the single amino acids ( $n=20$ parameters) and nearest- and next-nearest-neighbor ami-

242 no-acid pairs $\left(n=20^{2} \times 2=800\right)$ and the frequencies of CDR3 lengths ( $n=38$ for $\operatorname{lgH}$ and 26 for

243 TCR $\beta$ ), following previous reports (Mora et al., 2010). Descriptor models were trained on the

244 frequencies of the single amino acids ( $n=20$ parameters), the product of each pair of descriptors

245 at different positions $\left(n=5^{2}=25\right.$ per set), lengths, and, as indicated, on amino-acid frequencies

246 for the first- and last-four amino acids (roughly corresponding to the CDR3 stem or "torso"

247 (North et al., 2011; Finn et al., 2016); $n=20$ ), the product of each (non-redundant) pair of de-

248 scriptors at the same position $(n=(5 \times 4) / 2=10)$, the product of each pair of descriptors for the

249 stem ( $n=25$ per set), and the product of descriptors at each three $\left(n=5^{3}=125\right)$. For Test 3 , mod-

250 els were trained on the expectation values of each descriptor for the stem $(n=5)$ and full-length

251 CDR3 $(n=5)$, pairs of descriptors at the same position, cross-loop pairs, and nearest- and next-

252 nearest-neighbor pairs for the full-length CDR3 and the stem ( $n=25$ per set), anchoring se-

253 quences with an initial cysteine and terminal tryptophan for speed.

254 Fitting was performed using Metropolis-Hastings Markov-chain Monte Carlo sampling with the

255 acceptance criterion

$$
A\left(\sigma^{\prime}, \sigma\right)=\min \left(\frac{p\left(\sigma^{\prime}\right)}{p(\sigma)} \frac{g\left(\sigma^{\prime} \mid \sigma\right)}{g\left(\sigma \mid \sigma^{\prime}\right)}, 1\right)
$$

256 where $\sigma$ is the original sequence and $\sigma^{\prime}$ is proposed according to the proposal distribution

$257 g\left(\sigma \mid \sigma^{\prime}\right)$, updating biases via gradient descent using an adaptive step size, using an adaptive

258 burn-in period and autocorrelation time, and a time limit of 24 hours/fit as a stopping condition.

259 Each model was trained for 24 hours on 44 parallel CPUs using the National Science Founda-

260 tion's high-performance supercomputing cluster, XSEDE (Towns et al., 2014). To avoid overfit-

261 ting, we prohibited sample size from exceeding the size of the training set.

262 Probabilities. The probability of a sequence $\sigma$ according to a MaxEnt model $M$ was calculated as

$$
p(\sigma \mid M)=\frac{1}{Z} e^{-E_{\sigma \vee M}}
$$


Arora et al. (2019) Repertoire-Based Diagnostics Using Statistical Biophysics

263 where $E_{\sigma \mid M}$ is the energy of $\sigma$ and the normalization constant $Z=\sum_{\sigma} e^{-E_{\sigma \mid M}}$ was estimated via 264 bridge sampling (Meng and Wong, 1996; Gelman and Meng, 1998) using Harvard Medical

265 School's high-performance computing cluster.

266 Classification (Test 3). We fit descriptor models on each of the day 0/day 7 before-and-after

$267 \mathrm{IgG}^{+}$repertoire pairs ( $n=31: n=17$ from day 0 , including replicates, and $n=14$ day 7 ) from the in-

268 fluenza vaccination dataset (Vollmers et al., 2013; Arora et al., 2018) and used a support-

269 vector-machine (SVM) on the final models for classification (excluding length biases, which in-

270 teract with the normalization constant), using the median area under the receiver-operator-

271 characteristic curve (AUC/ROC) as the quality measure (taken over $n=10,000$ repeats; mean

272 preferred over median given the observed highly skew AUC distributions expected from strong

273 performance with outliers; Fig. 4 top inset), with stratified $k$-fold cross-validation (without over-

274 sampling; 17 vs. 14 was considered sufficiently balanced, but see null-model comparison below)

275 to avoid overfitting (for $k=2,3,5$, and 10 to confirm robustness) and comparison to the AUC of

276 randomly relabeled data as a null model (also $n=10,000$ repeats) to assess statistical signifi-

277 cance. Mann-Whitney $\cup p$-value was calculated to test that the two AUC distributions were dif-

278 ferent. The significance of the AUC was understood as the probability that it could arise from a

279 random classifier by chance; the $p$-value for significance of the AUC was therefore calculated as

280 the fraction of the area under the null-model distribution to the right of the AUC. Histograms

281 were plotted. All analyses were performed using Python's numpy and scipy libraries.

\section{Figure Legends}

283 Figure 1. MaxEnt Based on Amino Acids' Biophysical Properties. (a) Amino acids as vectors, 284 shown here as a heatmap, in a 5-dimensional descriptor space. (b) Amino acids with similar 285 properties lie near to each other in descriptor space. These similarities can be visualized by cal286 culating all pairwise Euclidean distances of the amino acids in descriptor space, constructing a

287 (complete, $K_{20}$ ) network with the amino acids as nodes and the distances as weighted edges, 288 and then for clarity keeping only edges with weights $\leq 1.1$. For example, aspartate (D) and glu289 tamate $(E)$ (red boxes in (a)) lie near to each other in descriptor space, illustrated by their similar 290 pattern in the heatmap (with prominent differences only in the dimension corresponding to de291 scriptor 4), and so are adjacent in the network. Amino acids are colored according to a familiar 292 groupings (basic, aliphatic, etc.) to demonstrate that their configuration in descriptor space 293 agrees with these groupings. (c) Data preparation and model training. Repertoires were first 294 split into training and test sets, and the features of the training set measured. Models were 
Arora et al. (2019) Repertoire-Based Diagnostics Using Statistical Biophysics

295

296

297

298

299

300

301

302

303

304

305

306

307

308

309

310

311

312

313

314

315

316

317

318

319

320

321

322

323

324

325

326

trained through iterative sampling, comparing of sample and observed features, and updating biases. (d) Example using a highly simplified toy repertoire consisting of a training set of two unique 3-amino-acid sequences, CTR and DVW (common in stems of IgH CDR3s). The models learn constraints that distinguish the training set from random 3-mers. For amino-acid models, constraints are the frequencies of letters; for descriptor models, constraints are expectation values of descriptors and descriptor products at given positions (here, nearest-neighbor pairs). Model output is shown in the last row. The descriptor model has learned the pattern of biophysical relationships, such that sequences that are biophysically similar to sequences in the training set also appear in the sample, albeit at lower frequency than the sequences in the training set.

Figure 2. Training and normalization. Distance (root-mean-squared error, RMSE) between training data and model sample as a function of iterations of model training. Data shown is for all models in Tests 1 and 2. Insets, bridge sampling for representative fits showing overlap between model- (blue) and randomly sampled sequences (gray).

Figure 3. Comparison of amino-acid vs. descriptor models. Head-to-head tests on lgH (left) and TCR $\beta$ (right) repertoires; the better performer is shaded green. (a) Validation comparison of models of IgH vs. TCR $\beta$ repertoires; IgH models strongly prefer lgH sequences (yellow) and TCR $\beta$ models strongly prefer TCR $\beta$ sequences (red; results shown are for the 325-parameter descriptor models). (b)-(d) Comparisons of an amino-acid model to a descriptor model, both trained/tested on the same training/test set. Density to the left of the vertical dashed line represents sequences for which the amino-acid model gave the higher probability; density to the right (filled) represents higher probability per the descriptor model. Vertical red lines denote medians of the probability densities. (b) Test 1 : models fitting similar positional information (single positions plus nearest- and next-nearest neighbors); amino-acid models perform better. (c) Test 2: models fitting similar numbers of parameters (420 non-length parameters for the amino-acid model vs. 325 for the descriptor model); descriptor models perform better. (d) Test 2, continued: amino-acid benchmark model (820 parameters; nearest- and next-nearest neighbors) vs. the descriptor model in (c); descriptor models perform better.

Figure 4. Classification of pre- vs. post-flu vaccination in human subjects. Shown is the median AUC (red) for 10,000 training-test splits using stratified 3-fold cross-validation of an SVM on 31 pre- and post-vaccination samples from the same subjects. Insets show the distributions of AUCs from all 10,000 splits of the real data (blue) and from 10,000 splits in which the data was randomly relabeled, to measure the probability that the median performance could have been 
bioRxiv preprint doi: https://doi.org/10.1101/519108; this version posted January 13,2019 . The copyright holder for this preprint (which was not certified by peer review) is the author/funder, who has granted bioRxiv a license to display the preprint in perpetuity. It is made available under aCC-BY-NC 4.0 International license.

\section{Arora et al. (2019) Repertoire-Based Diagnostics Using Statistical Biophysics}

327 the result of chance (gray). Red, median. The p-value is the area in the random-relabeling dis328 tribution to the right of the median. 
bioRxiv preprint doi: https://doi.org/10.1101/519108; this version posted January 13,2019 . The copyright holder for this preprint (which was not certified by peer review) is the author/funder, who has granted bioRxiv a license to display the preprint in perpetuity. It is made available under aCC-BY-NC 4.0 International license.

Arora et al. (2019) Repertoire-Based Diagnostics Using Statistical Biophysics

Figure 1
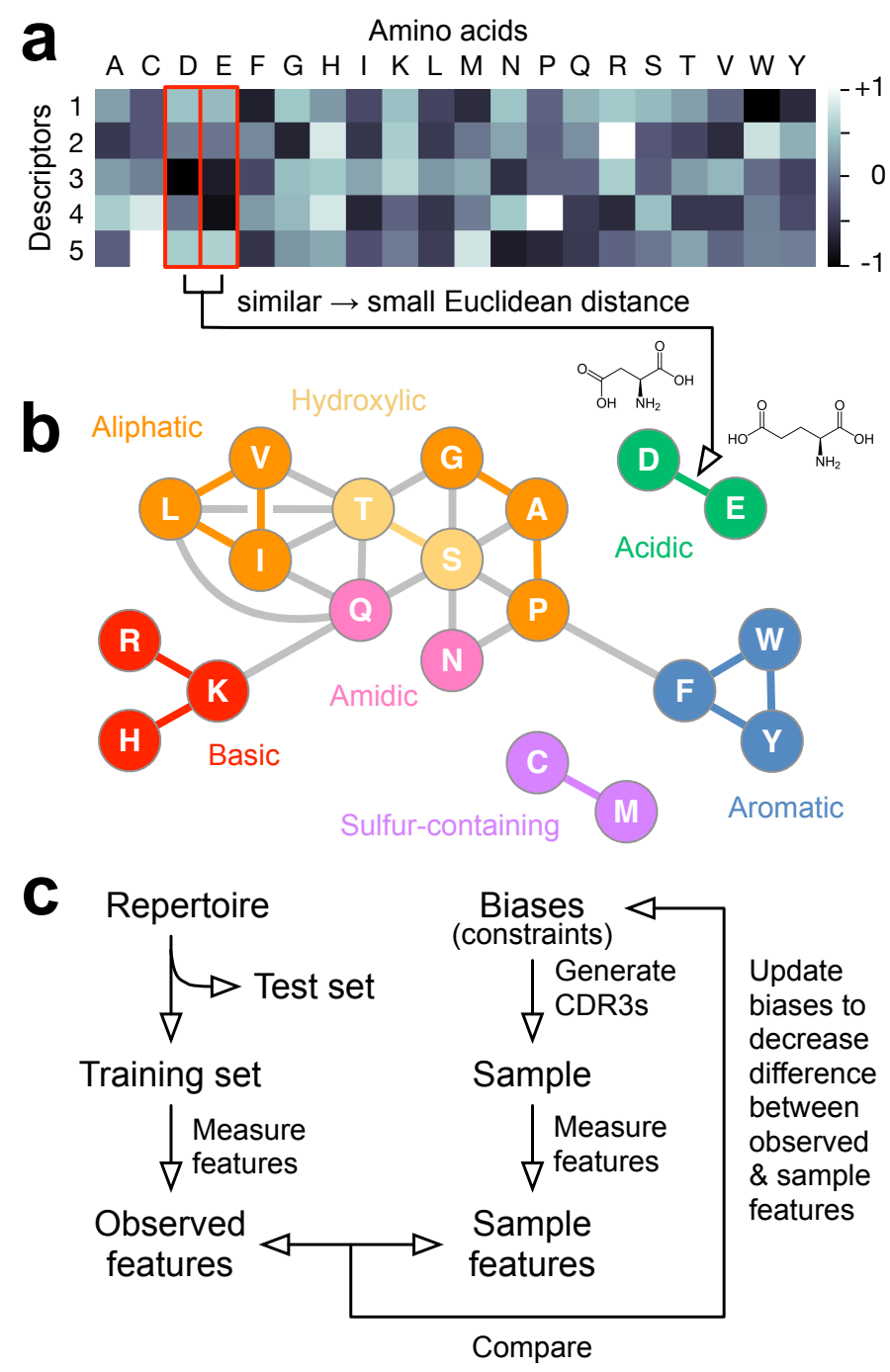

\begin{tabular}{|c|c|c|}
\hline Model & Amino acids & Descriptors \\
\hline Training set & 50:50 CTR:DVW & 50:50 CTR:DVW \\
\hline Features & $\begin{array}{c}\text { Amino acid } \\
\text { frequencies }(n=420)\end{array}$ & $\begin{array}{l}\text { Descriptor expecta- } \\
\text { tion values }(n=30)\end{array}$ \\
\hline
\end{tabular}

Constraints Sequences are equal Sequences are equal which biases parts C, D, R, T, V, parts $\bullet, 0,0, \bullet, \bullet$, will learn and $W$ and equal parts CT, TR, DV, and and equal and VW parts $\boldsymbol{O}, \boldsymbol{O}$, and $\boldsymbol{O}$
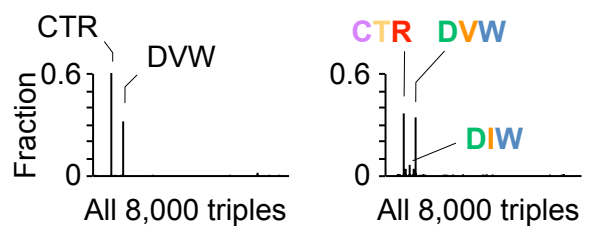
bioRxiv preprint doi: https://doi.org/10.1101/519108; this version posted January 13,2019 . The copyright holder for this preprint (which was not certified by peer review) is the author/funder, who has granted bioRxiv a license to display the preprint in perpetuity. It is made available under aCC-BY-NC 4.0 International license.

\section{Arora et al. (2019) Repertoire-Based Diagnostics Using Statistical Biophysics}

Figure 2

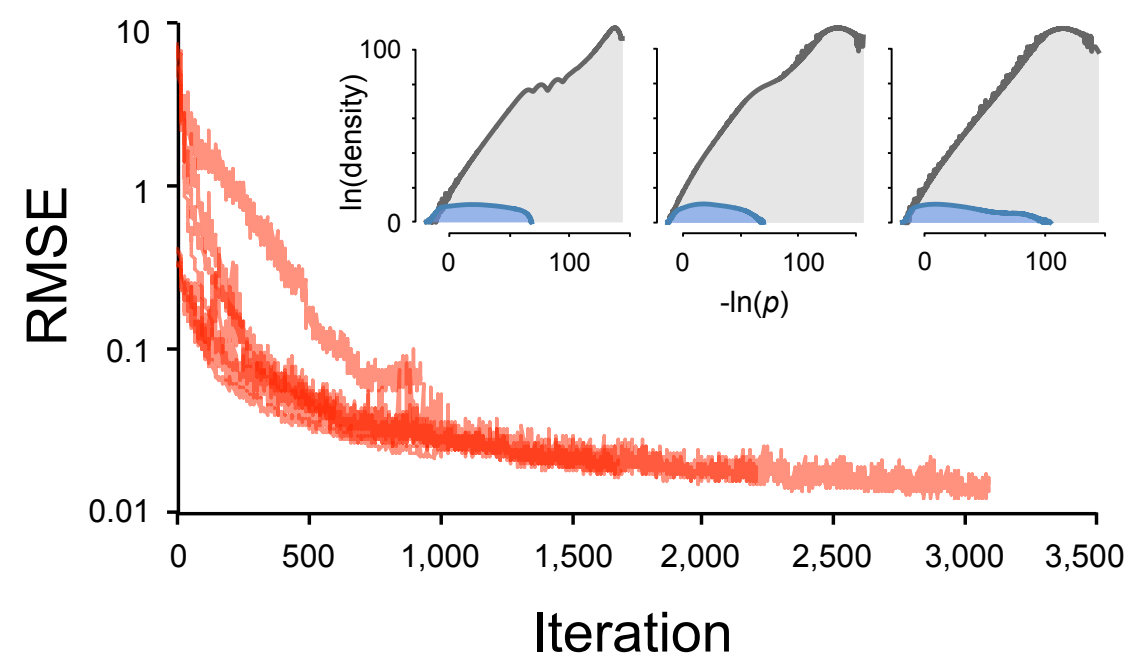


bioRxiv preprint doi: https://doi.org/10.1101/519108; this version posted January 13,2019 . The copyright holder for this preprint (which was not certified by peer review) is the author/funder, who has granted bioRxiv a license to display the preprint in perpetuity. It is made available under aCC-BY-NC 4.0 International license.

\section{Arora et al. (2019) Repertoire-Based Diagnostics Using Statistical Biophysics}

$\lg \mathrm{H}$ test sequences
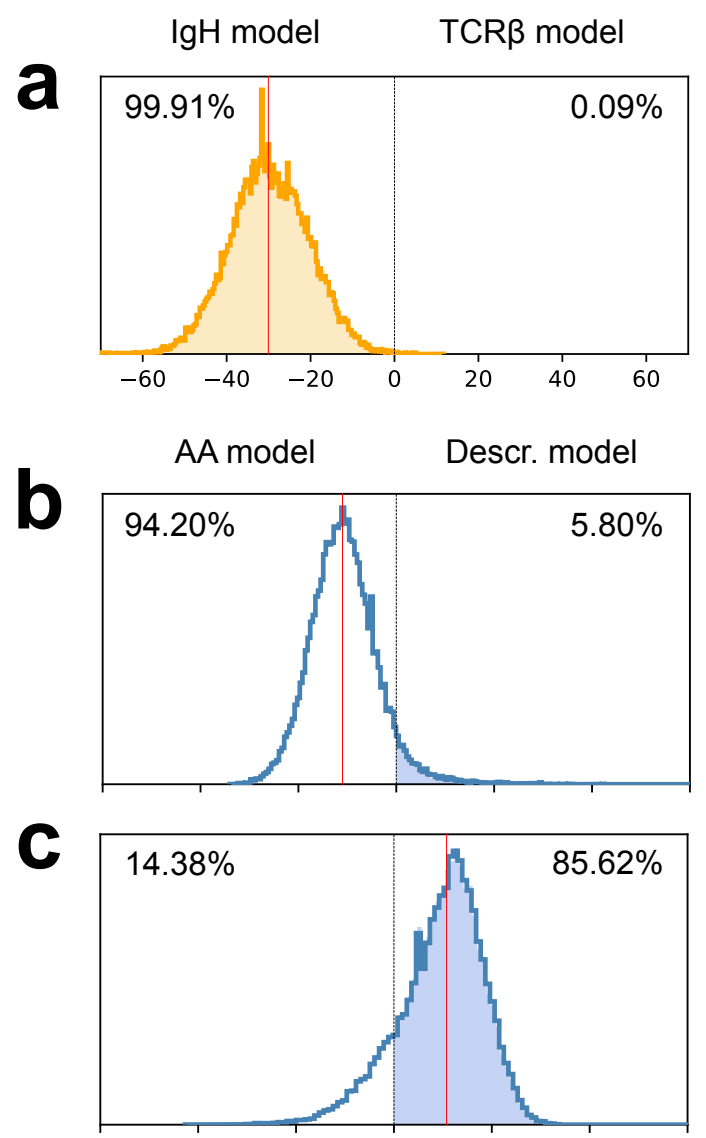

d

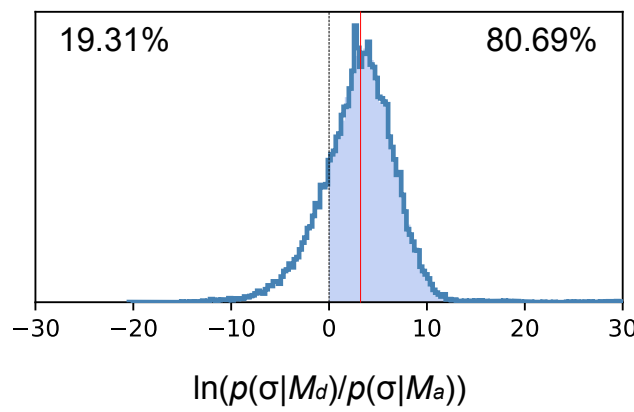

TCR $\beta$ test sequences

IgH model TCR $\beta$ model

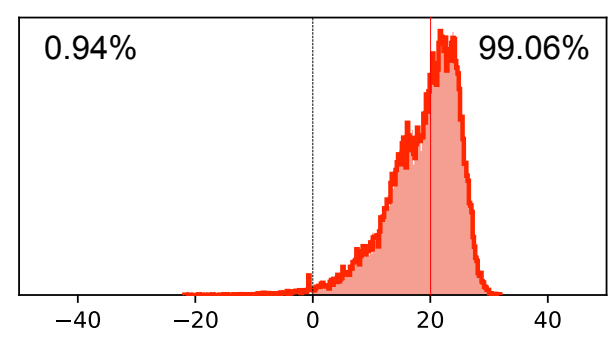

AA model Descr. model
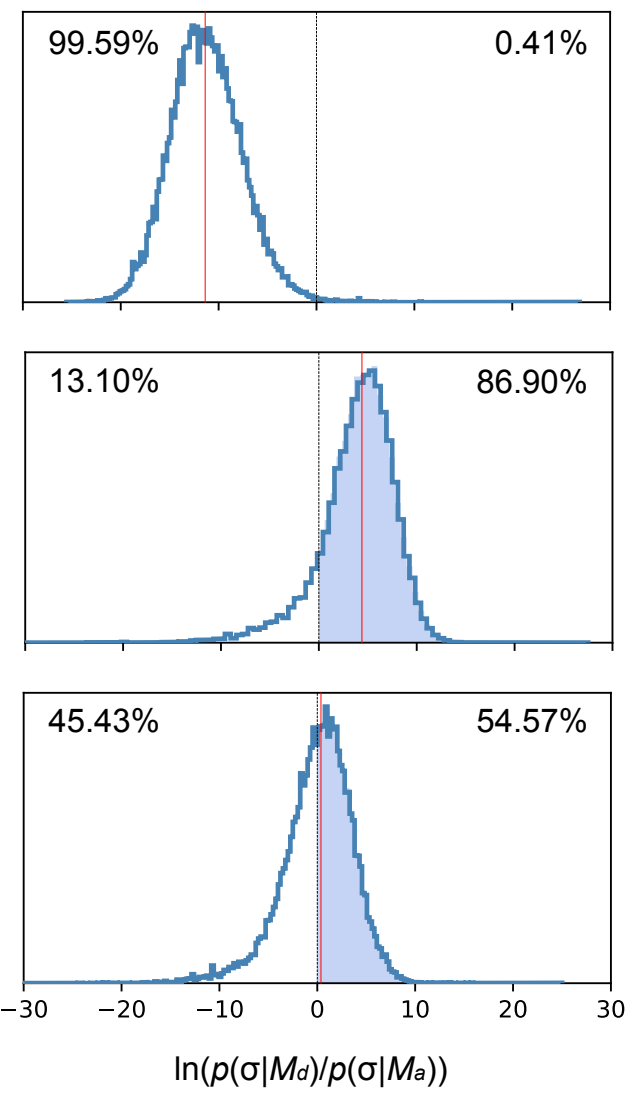
bioRxiv preprint doi: https://doi.org/10.1101/519108; this version posted January 13,2019 . The copyright holder for this preprint (which was not certified by peer review) is the author/funder, who has granted bioRxiv a license to display the preprint in perpetuity. It is made available under aCC-BY-NC 4.0 International license.

Arora et al. (2019) Repertoire-Based Diagnostics Using Statistical Biophysics

Figure 4

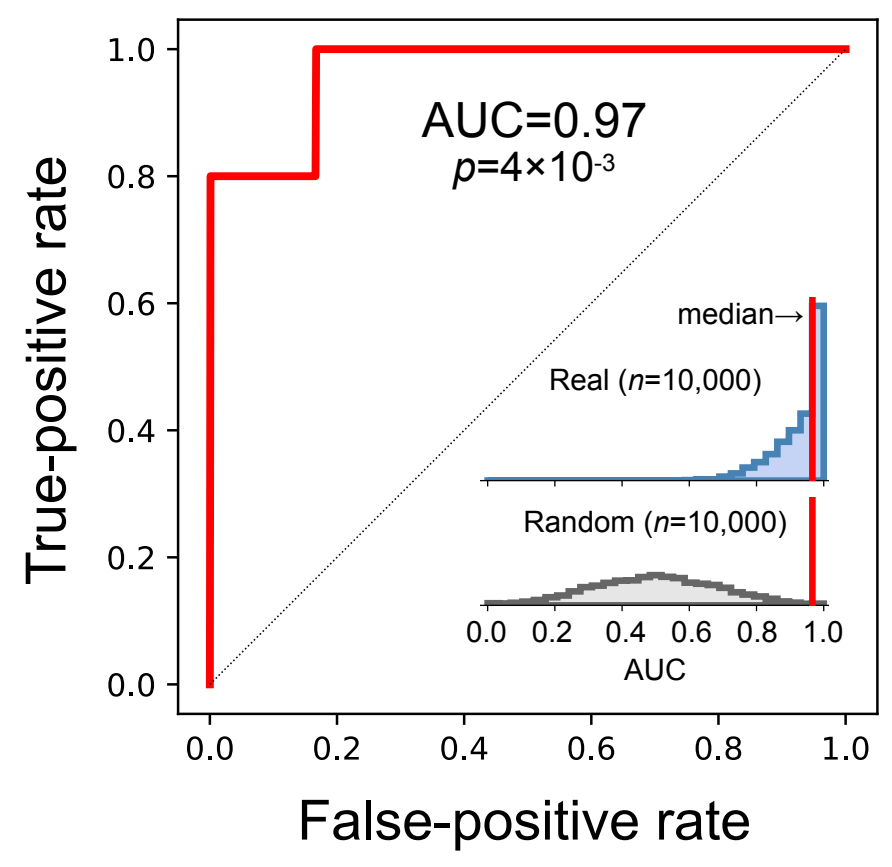


Arora et al. (2019) Repertoire-Based Diagnostics Using Statistical Biophysics

337

338

339

340

341

342

343

344

345

346

347

348

349

350

351

352

353

354

355

356

357

358

359

360

361

362

363

\section{References}

Arnaout, R., Lee, W., Cahill, P., Honan, T., Sparrow, T., Weiand, M., Nusbaum, C., Rajewsky, K., and Koralov, S.B. (2011). High-Resolution Description of Antibody Heavy-Chain Repertoires in Humans. PLOS ONE 6, e22365.

Arora, R., Burke, H.M., and Arnaout, R. (2018). Immunological Diversity with Similarity. BioRxiv 483131.

Bialek, W., and Ranganathan, R. (2007). Rediscovering the power of pairwise interactions. ArXiv:0712.4397 [q-Bio].

Buck, C.A. (1992). Immunoglobulin superfamily: structure, function and relationship to other receptor molecules. Semin. Cell Biol. 3, 179-188.

DeWitt, W.S., Lindau, P., Snyder, T.M., Sherwood, A.M., Vignali, M., Carlson, C.S., Greenberg, P.D., Duerkopp, N., Emerson, R.O., and Robins, H.S. (2016). A Public Database of Memory and Naive B-Cell Receptor Sequences. PLoS ONE 11, e0160853.

Dosztányi, Z., and Torda, A.E. (2001). Amino acid similarity matrices based on force fields. Bioinformatics $17,686-699$.

Emerson, R.O., DeWitt, W.S., Vignali, M., Gravley, J., Hu, J.K., Osborne, E.J., Desmarais, C., Klinger, M., Carlson, C.S., Hansen, J.A., et al. (2017). Immunosequencing identifies signatures of cytomegalovirus exposure history and HLA-mediated effects on the T cell repertoire. Nat. Genet. 49, 659-665.

Finn, J.A., Koehler Leman, J., Willis, J.R., Cisneros, A., Crowe, J.E., and Meiler, J. (2016). Improving Loop Modeling of the Antibody Complementarity-Determining Region 3 Using Knowledge-Based Restraints. PLoS ONE 11, e0154811.

Gelman, A., and Meng, X.-L. (1998). Simulating Normalizing Constants: From Importance Sampling to Bridge Sampling to Path Sampling. Statistical Science 13, 163-185.

361 Greiff, V., Weber, C.R., Palme, J., Bodenhofer, U., Miho, E., Menzel, U., and Reddy, S.T. (2017). Learning the High-Dimensional Immunogenomic Features That Predict Public and Private Antibody Repertoires. J. Immunol. 199, 2985-2997. 
Arora et al. (2019) Repertoire-Based Diagnostics Using Statistical Biophysics

364

365

366

367

368

369

370

371

372 Kim, S., Thiessen, P.A., Bolton, E.E., Chen, J., Fu, G., Gindulyte, A., Han, L., He, J., He, S.,

373 Shoemaker, B.A., et al. (2016). PubChem Substance and Compound databases. Nucleic Acids

374 Res. 44, D1202-1213.

375 Lefranc, M.P., Giudicelli, V., Ginestoux, C., Bodmer, J., Müller, W., Bontrop, R., Lemaitre, M., 376 Malik, A., Barbié, V., and Chaume, D. (1999). IMGT, the international ImMunoGeneTics data377 base. Nucleic Acids Res. 27, 209-212.

378 Marks, D.S., Colwell, L.J., Sheridan, R., Hopf, T.A., Pagnani, A., Zecchina, R., and Sander, C. 379 (2011). Protein 3D Structure Computed from Evolutionary Sequence Variation. PLOS ONE 6, 380

Hellberg, S., Sjöström, M., Skagerberg, B., and Wold, S. (1987). Peptide quantitative structureactivity relationships, a multivariate approach. J. Med. Chem. 30, 1126-1135.

Jiang, N., He, J., Weinstein, J.A., Penland, L., Sasaki, S., He, X.-S., Dekker, C.L., Zheng, N.-Y., Huang, M., Sullivan, M., et al. (2013). Lineage structure of the human antibody repertoire in response to influenza vaccination. Sci Transl Med 5, 171ra19.

Kaplinsky, J., Li, A., Sun, A., Coffre, M., Koralov, S.B., and Arnaout, R. (2014). Antibody repertoire deep sequencing reveals antigen-independent selection in maturing B cells. Proc. Natl. Acad. Sci. U.S.A. 111, E2622-2629. e28766.

Meng, X.-L., and Wong, W.H. (1996). Simulating ratios of normalizing constants via a simple identity: A theoretical exploration. Statistica Sinica 6, 831-860.

3 Mora, T., Walczak, A.M., Bialek, W., and Callan, C.G. (2010). Maximum entropy models for antibody diversity. PNAS 107, 5405-5410.

85 North, B., Lehmann, A., and Dunbrack, R.L. (2011). A new clustering of antibody CDR loop conformations. J. Mol. Biol. 406, 228-256.

87 Ostmeyer, J., Christley, S., Rounds, W.H., Toby, I., Greenberg, B.M., Monson, N.L., and Cowell, L.G. (2017). Statistical classifiers for diagnosing disease from immune repertoires: a case study using multiple sclerosis. BMC Bioinformatics 18, 401. 
Arora et al. (2019) Repertoire-Based Diagnostics Using Statistical Biophysics

390

391

392

393

394

395

396

397

398

399

400

401

402

403

404

405

406

407

408

409

410 Sun, Y., Best, K., Cinelli, M., Heather, J.M., Reich-Zeliger, S., Shifrut, E., Friedman, N., Shawe-

411 Taylor, J., and Chain, B. (2017). Specificity, Privacy, and Degeneracy in the CD4 T Cell Recep-

412 tor Repertoire Following Immunization. Front. Immunol. 8.

413 Susko, E., and Roger, A.J. (2007). On reduced amino acid alphabets for phylogenetic inference.

414 Mol. Biol. Evol. 24, 2139-2150.

415 Towns, J., Cockerill, T., Dahan, M., Foster, I., Gaither, K., Grimshaw, A., Hazlewood, V., Lath-

416 rop, S., Lifka, D., Peterson, G.D., et al. (2014). XSEDE: Accelerating Scientific Discovery. Com-

417 puting in Science \& Engineering 16, 62-74. 
Arora et al. (2019) Repertoire-Based Diagnostics Using Statistical Biophysics

418 Vollmers, C., Sit, R.V., Weinstein, J.A., Dekker, C.L., and Quake, S.R. (2013). Genetic meas-

419 urement of memory B-cell recall using antibody repertoire sequencing. Proc. Natl. Acad. Sci.

420 U.S.A. 110, 13463-13468.

421 Walter, K.U., Vamvaca, K., and Hilvert, D. (2005). An active enzyme constructed from a 9-

422 amino acid alphabet. J. Biol. Chem. 280, 37742-37746.

423 Weitzner, B.D., Dunbrack, R.L., and Gray, J.J. (2015). The origin of CDR H3 structural diversity. 424 Structure 23, 302-311.

425 Yeo, G., and Burge, C.B. (2004). Maximum entropy modeling of short sequence motifs with ap426 plications to RNA splicing signals. J. Comput. Biol. 11, 377-394. 